\title{
SLOCC Invariants for Multipartite Mixed States
}

\author{
Naihuan Jing ${ }^{1,5} *$ Ming Li ${ }^{2,3}$, Xianqing Li-Jost ${ }^{2}$, Tinggui Zhang ${ }^{2}$, and Shao-Ming Fei ${ }^{2,4}$ \\ ${ }^{1}$ School of Sciences, South China University of Technology, Guangzhou 510640, China \\ ${ }^{2}$ Max-Planck-Institute for Mathematics in the Sciences, 04103 Leipzig, Germany \\ ${ }^{3}$ Department of Mathematics, China University of Petroleum, Qingdao 266555, China \\ ${ }^{4}$ School of Mathematical Sciences, Capital Normal University, Beijing 100048, China \\ ${ }^{5}$ Department of Mathematics, North Carolina State University, Raleigh, NC 27695, USA

\begin{abstract}
We construct a nontrivial set of invariants for any multipartite mixed states under the SLOCC symmetry. These invariants are given by hyperdeterminants and independent from basis change. In particular, a family of $d^{2}$ invariants for arbitrary $d$-dimensional even partite mixed states are explicitly given.
\end{abstract}

PACS numbers: 03.67.-a, 02.20.Hj, 03.65.-w

*Electronic address: jing@math.ncsu.edu 


\section{INTRODUCTION}

Classification of multipartite states under stochastic local operations and classical communication (SLOCC) has been a central problem in quantum communication and computation. Recently advances have been made for the classification of pure multipartite states under SLOCC [1, 2] and the dimension of the space of homogeneous SLOCC-invariants in a fixed degree is given as a function of the number of qudits. In this work we present a general method to construct polynomial invariants for mixed states under SLOCC. In particular we also derive general invariants under local unitary (LU) symmetry for mixed states.

Polynomial invariants have been investigated in [3-6], which allow in principle to determine all the invariants of local unitary transformations. However, in practice it is a daunting task to solve the large system of algebraic equations. Moreover, little is known for mixed multipartite states even for $2 \times 2 \times 2$-systems. In [7] a complete set of 18 polynomial invariants is presented for the locally unitary equivalence of two qubit-mixed states. Partial results have been obtained for three qubits states [8], tripartite pure and mixed states [9], and some generic mixed states [10 12]. Recently the local unitary equivalence problem for multiqubit [13] and general multipartite [14] pure states have also been solved.

In general there are limited tools available to resolve the SLOCC symmetry for mixed multipartite states. Ideally it is hoped that a complete set of invariants under local unitary transformations can be found. Nevertheless usually these invariants depend on the detailed expressions of pure state decompositions of a state. For a given state, such pure state decompositions are infinitely many. Particularly when the density matrices are degenerate, the problem becomes more complicated.

In this paper, we present a general method to construct non-trivial invariants under SLOCC symmetry group. All invariants found using this method are a priori LU-invariants, as the LU symmetry group is contained in the SLOCC symmetry group. Therefore SLOCCinvariants are generally much fewer than LU-invariants. Very few information is known about the SLOCC-invariants except for that the degree of the invariant subring [1], and even less is known about how to systematically construct the invariants. We will give a general method to construct four families of SLOCC-invariants for any mixed state of even-partite plus an iterative method to construct general fundamental invariants in all dimensions.

We show that the Cayley's two hyperdeterminants [15, 16] can be used to construct such 
invariants. Cayley's second hyperdeterminant has been used to study the entanglement measure like concurrence [17-21] and 3-tangles [22]. It also played an important role in classification of multipartite pure states [23 25]. In [26] we proved that the hyperdetermiants give LU-invariants that are independent from the pure state decomposition and base transition. One of our current main results is that the generalized characteristic polynomials give actually SLOCC invariants and we further show that in the even partite cases the Cayley's first hyperdeterminant also lead to some of the nontrivial SLOCC invariants for mixed states.

\section{HYPER-MATRIX REPRESENTATIONS OF MIXED STATES}

Let $H$ be the state space of the mixed multipartite quantum state: $H=H_{1} \otimes H_{2} \otimes \cdots \otimes H_{n}$, where $H_{k}$ is a Hilbert space of dimension $d_{k}$ with inner product $\langle,\rangle_{i}$. Suppose $\sigma_{i}^{(k)}(i=$ $\left.0, \ldots, d_{k}^{2}-1\right)$ are orthonormal basis of hermitian operators in $\operatorname{End}\left(H_{i}\right)$. Here we can take the Gell-Mann basis, where $\sigma_{0}^{(i)}=I$ is the identity. In particular, they are Pauli spin matrices when $d_{i}=2$.

Then $\rho$ can be expressed as

$$
\rho=\sum_{i_{1} \cdots i_{n}} a_{i_{1} \cdots i_{n}} \sigma_{i_{1}}^{(1)} \otimes \cdots \otimes \sigma_{i_{n}}^{(n)}
$$

where the $n$-dimensional matrix or hypermatrix $A=\left(a_{i_{1} \cdots i_{n}}\right)$ is called the matrix represen-

tation of $\rho$ with respect to the bases $\left\{\sigma_{i}^{(k)}\right\}$. Here the format of the matrix $A$ is $d_{1}^{2} \times \cdots \times d_{n}^{2}$, and usual rectangular matrices of size $m \times n$ are 2 -dimensional matrices of format $m \times n$. One can use $A=[\rho]_{\sigma}$ to denote the matrix representation of $\rho$ with respect to the orthonormal bases $\sigma=\left\{\sigma_{i}^{(k)}\right\}$. Sometimes the reference to $\sigma$ is omitted if it is clear from the context.

We recall the multiplication of the hyper-matrix $A$ of format $f_{1} \times \cdots \times f_{n}$ by a $f_{i} \times f_{i}$-matrix $B$ as follows:

$$
B *_{k} A=C,
$$

where

$$
C_{i_{1} \cdots i_{n}}=\sum_{j=1}^{f_{k}} b_{i_{k} j} a_{i_{1} \cdots i_{k-1} j i_{k+1} \cdots i_{n}} .
$$

This generalizes the notion of matrix multiplication. When $A$ is a regular rectangular matrix, then $B *_{1} A=B A, B *_{2} A=A B^{t}$ and $\left(B *_{1} C *_{2}\right) A=B A C^{t}$, where $t$ stands for transpose. 
It is easy to see that the matrix representation of $\rho$ under basis change from $\sigma$ to $\sigma^{\prime}$ can be simply described by

$$
[\rho]_{\sigma^{\prime}}=\left(P_{1} *_{1} \cdots P_{n} *_{n}\right)[\rho]_{\sigma}
$$

where the unitary matrix $P_{k}$ is the transition matrix from the orthonormal basis $\left\{\sigma_{i}^{(k)}\right\}$ to the orthonormal basis $\left\{\sigma_{i}^{\prime(k)}\right\}$ for $k=1, \cdots, n$.

A function $F(\rho)$ is invariant under SLOCC transformation if $F(\rho)=F\left(A \rho A^{\dagger}\right)$ for any invertible $A=A_{1} \otimes \cdots \otimes A_{n} \in G=S L\left(d_{1}\right) \otimes \cdots \otimes S L\left(d_{n}\right)$, the SLOCC symmetry group, where $\dagger$ denote transpose and conjugate. A true SLOCC-invariant should only depend on $\rho$, i.e., an invariant $F(\rho)$ given in terms of the matrix $[\rho]$ should be independent from the matrix representation [26]. Since our matrix representation is given as an un-normalized form, the SLOCC-invariants can be taken as a relative invariant under the larger group $\hat{G}=G L\left(d_{1}\right) \otimes \cdots \otimes G L\left(d_{n}\right)$, or a projective invariant under $\hat{G}$.

Two hypermatrices of the same format can be added together to give rise to a third hypermatrix by $\left(a_{i_{1} \cdots i_{n}}\right)+\left(b_{i_{1} \cdots i_{n}}\right)=\left(a_{i_{1} \cdots i_{n}}+b_{i_{1} \cdots i_{n}}\right)$. The scalar product of a hypermatrix by a number is defined as usual. Then the set of hypermatrices of format $f_{1} \times \cdots \times f_{n}$ forms a vector space of dimension $f_{1} \cdots f_{n}$. We denote the vector space by $\operatorname{Mat}\left(f_{1}, \cdots, f_{n}\right)$.

We consider invariants under the SLOCC symmetry. Let us first look at the special example of bipartite case to motivate our later discussion. Suppose $A$ is the (square) matrix representation of the mixed state $\rho$ on $H^{\otimes 2}$. Using the same method of [26] it is easy to see the following result.

Proposition 1 Suppose $A$ is the Bloch matrix representation of the bipartite mixed state $\rho$ on $\left(\mathbb{C}^{d}\right)^{\otimes 2}$. Then the coefficients $F_{i}(A), i=1,2, \ldots, d^{2}$, of the characteristic polynomials of A:

$$
\begin{aligned}
\operatorname{det}(\lambda I-A) & =\lambda^{d}-\operatorname{Tr}(A) \lambda^{d-1}+\cdots+(-1)^{d} \operatorname{det}(A) \\
& =\sum_{i=0}^{d} \lambda^{d-i} F_{i}(A)
\end{aligned}
$$

are LU-invariants. In particular, $\operatorname{tr}(A)$ and $\operatorname{det}(A)$ are $L U$-invariants of the two-partite state $\rho$.

We remark that when $\rho$ is a bipartite mixed state in arbitrary dimensional space $H_{1} \otimes$ $H_{2}$, then the Bloch matrix representation $A$ is a rectangular matrix. Then $\operatorname{det}\left(A A^{T}\right)$ and 
$\operatorname{det}\left(A^{T} A\right)$ are LU-invariants. When $m \neq n$, it seems that these are the only known SLOCCinvariants according to [1].

For pure states $|\phi\rangle=\sum_{i_{1}, \cdots, i_{n}} a_{i_{1} \cdots i_{n}}\left|i_{1} \cdots i_{n}\right\rangle$, we can associate the hypermatrix $A(|\phi\rangle)=$ $\left(a_{i_{1} \cdots i_{n}}\right)$, then the format will be simpler. For bipartite pure state $|\phi\rangle$, the determinant $\operatorname{det}(|\phi\rangle)$ is clearly a SLOCC-invariant.

In the following we extend the result in Proposition 1 to show that $F_{i}(A)$ are in fact SLOCC-invariants.

\section{HYPERDETERMINANTS AND SLOCC-INVARIANTS}

Given any hypermatrix $A$, one defines the associated multilinear form $f_{A}: H_{1} \otimes \cdots \otimes H_{n} \mapsto$ $\mathbb{C}$ given by

$$
f_{A}\left(x^{(1)}, \cdots, x^{(n)}\right)=\sum_{i_{1}, \cdots, i_{n}} a_{i_{1} \cdots i_{n}} x_{i_{1}}^{(1)} \cdots x_{i_{n}}^{(n)} .
$$

The multilinear form $f$ can also be written as a tensor in $H_{1}^{*} \otimes \cdots \otimes H_{n}^{*}$ :

$$
f_{A}=\sum_{i_{1}, \cdots, i_{n}} a_{i_{1} \cdots i_{n}} f_{i_{1}}^{(1)} \otimes \cdots \otimes f_{i_{n}}^{(n)}
$$

where $f_{i}^{(k)}$ are standard 1-forms on $H_{k}$ such that $f_{i}^{(k)}\left(e_{j}^{(k)}\right)=\delta_{i j}$ for $i, j=1, \cdots, \operatorname{dim}\left(V_{i}\right)$.

To examine the action of the SLOCC-symmetry on the hypermatrix representation of $\rho$, we first give the following important lemma.

Lemma 2 For fixed $A \in M_{m}$ and $C \in M_{n}$, let $\phi: M_{m, n} \longrightarrow M_{m, n}$ be the linear map defined by $B \mapsto A B C$. Then $\operatorname{det}(\phi)=\operatorname{det}(A)^{n} \operatorname{det}(C)^{m}$.

Proof: Choose a basis $B_{1}, \cdots, B_{m n}$ in $M_{m, n}$. Recall the vector realignment $v(B) \equiv \operatorname{vec}(B)$ [27]. For any rectangular matrix $B=\left(b_{i j}\right) \in M_{m, n}$, the column vector realignment is given by $v(B)=\left[b_{11}, \ldots, b_{m 1}, \cdots, b_{1 n}, \ldots, b_{m n}\right]^{t}$. The vector realignment satisfies the following fundamental property [27]:

$$
v(A B C)=\left(C^{t} \otimes A\right) v(B)
$$

where $C^{t} \otimes A$ is the Kronecker tensor product. It is also clear that $v\left(B_{1}\right), \cdots, v\left(B_{m n}\right)$ form a basis of $\mathbb{C}^{m n}$. Therefore the map $\phi$ induces an isomorphic linear operator on $M_{m n} \simeq \mathbb{C}^{m n}$ by $\phi(v(B))=v(A B C)$ as $v(\cdot)$ is apparently linear. We will denote the isomorphic map by 
the same symbol $\phi$. It then follows that

$$
\begin{aligned}
\phi\left[v\left(B_{1}\right), \cdots, v\left(B_{m n}\right)\right] & =\left[v\left(A B_{1} C\right), \cdots, v\left(A B_{m n} C\right)\right] \\
& =\left(C^{t} \otimes A\right)\left[v\left(B_{1}\right), \cdots, v\left(B_{m n}\right)\right]
\end{aligned}
$$

which means that $C^{t} \otimes A$ is the matrix of the linear operator $\phi \in \operatorname{End}\left(\mathbb{C}^{m n}\right)$. Hence $\operatorname{det}(\phi)=\operatorname{det}\left(C^{t} \otimes A\right)=\operatorname{det}(C)^{m} \operatorname{det}(A)^{n}$.

Consider a SLOCC symmetry $g=A_{1} \otimes \cdots \otimes A_{n} \in G=\operatorname{SL}\left(d_{1}\right) \otimes \cdots \otimes \operatorname{SL}\left(d_{n}\right)$ on the state space $H=H_{1} \otimes \cdots \otimes H_{n}$. This action is given by $\rho \mapsto g \rho g^{\dagger}=\left(A_{1} \otimes \cdots \otimes A_{n}\right) \rho\left(A_{1}^{\dagger} \otimes \cdots \otimes A_{n}^{\dagger}\right)$. Let $B_{k}$ be the matrix of the action of $A_{k}$ on the orthonormal basis $\left\{\sigma_{i}^{(k)}\right\}$,

$$
A_{k} \sigma_{i}^{(k)} A_{k}^{\dagger}=\sum_{j=1}^{d_{k}^{2}} B_{k}^{i j} \sigma_{i}^{(k)}
$$

then the hypermatrix of $g \rho g^{\dagger}$ under the action of $g \in G$ is seen to be

$$
\left[g \rho g^{\dagger}\right]=\left(B_{1} *_{1} \cdots *_{n-1} B_{n} *_{n}\right)[\rho]
$$

One usually writes it as $\left(B_{1} \otimes \cdots \otimes B_{n}\right)[\rho]$ when the hypermatrix $[\rho]$ is identified with its associated tensor via (6). This shows that the action of the SLOCC-symmetry on the mixed state $\rho$ is represented by similar hypermatrix multiplications when one introduces the matrix $B_{k}$ through (8) for each individual action of $A_{k}$ of the local SLOCC-symmetry $g=A_{1} \otimes \cdots \otimes A_{n}$. The great advantage of (9) is the similarity with the basis transition (3). The following result is immediate from Lemma 2 and the action (9).

Theorem 3 The action of SLOCC-group $\mathrm{SL}\left(d_{1}\right) \otimes \cdots \otimes \mathrm{SL}\left(d_{n}\right)$ on $\rho$ induces an action of $\mathrm{SL}\left(d_{1}^{2}\right) \times \cdots \times \mathrm{SL}\left(d_{n}^{2}\right)$ on its hypermatrix representation $[\rho]$.

We comment that a similar result also holds for a larger symmetry group $\widehat{S G}=\{g=$ $\left.A_{1} \otimes \cdots \otimes A_{n} \mid \operatorname{det}(g)=1, A_{k} \in \mathrm{GL}\left(d_{k}\right)\right\}$ when all $d_{i}$ are equal.

Theorem 3 translates the problem of SLOCC-invariants of $\rho$ into a specific problem of invariants of its hypermatrix representation $[\rho]$ under the group $\mathrm{SL}\left(d_{1}^{2}\right) \times \cdots \times \mathrm{SL}\left(d_{n}^{2}\right)$. This is exactly the classical problem studied long ago by Cayley.

Cayley [15] developed the theory of hyperdeterminant as the theory of homogeneous polynomials in matrix elements $A_{i_{1} \cdots i_{n}}$. A hyperdeterminant is a special homogeneous polynomial invariant under the action of $\left.\mathrm{SL}\left(n_{1}\right) \times \cdots \times \mathrm{SL}\left(n_{1}\right)\right)$. Modern account of Cayley's 
theory is the beautiful monograph [16]. Cayley studied two types of hyperdeterminants, and each is used in our current work. The Cayley's (second) hyperdeterminant $\operatorname{Det}(A)$ is defined as the resultant of the multilinear form $f_{A}$, that is, $\operatorname{Det}(A)$ is certain polynomial in components of the tensor $f_{A}$ which is zero if and only if the map $f_{A}$ has a non-trivial point where all partial derivatives with respect to the components of its vector arguments vanish (a non-trivial point means that none of the vector arguments are zero). For example, the resultant of $a_{1} x_{1}^{2}+a_{2} x_{1} x_{2}+a_{2} x_{2}^{2}=0$ is the polynomial $a_{2}^{2}-4 a_{1} a_{2}$.

It is known that the hyperdeterminant $\operatorname{Det}(A)$ exists for a given format and is unique up to a scalar factor provided that any factor in the format is less than or equal to the sum of the other factors in the format. Cayley's two hyperdeterminants are all invariant under the group $\mathrm{SL}\left(f_{1}\right) \otimes \cdots \otimes \mathrm{SL}\left(f_{n}\right)$. In fact the hyperdeterminant Det satisfies the following multiplicative property. Suppose $A$ is a hypermatrix of format $f_{1} \times \cdots \times f_{n}$ and $B$ is any $f_{i} \times f_{i}$ matrix, then

$$
\operatorname{Det}\left(B *_{i} A\right)=\operatorname{Det}(A) \operatorname{det}(B)^{N / f_{i}}
$$

where $N$ is the degree of $\operatorname{Det}(A)$. Therefore we have the following result.

Theorem 4 The hyperdeterminant of the matrix of $\rho$ is a relative invariant under the action of $\mathrm{GL}\left(d_{1}\right) \otimes \cdots \otimes \mathrm{GL}\left(d_{n}\right)$, thus invariant under the $S L O C C$ symmetry group $G=\mathrm{SL}\left(d_{1}\right) \otimes$ $\cdots \otimes \operatorname{SL}\left(d_{n}\right)$.

For an even dimensional matrix $A=\left(A_{i_{1} \cdots i_{2 n}}\right)$, where $1 \leq i_{1}, \ldots, i_{2 n} \leq N$, the Cayley's first hyperdeterminant $h \operatorname{det}(A)$ is the following polynomial

$$
h \operatorname{det}(A)=\frac{1}{N !} \sum_{\sigma_{1}, \cdots, \sigma_{2 n} \in S_{N}} \operatorname{sgn}(\sigma) \prod_{i=1}^{N} A_{\sigma_{1}(i), \ldots, \sigma_{2 n}(i)},
$$

where $\operatorname{sgn}(\sigma)=\prod_{i=1}^{n} \operatorname{sgn}\left(\sigma_{i}\right)$. The hyperdeterminant hdet reduces to the usual determinant when $A$ is a square matrix.

Cayley's second hyperdeterminant [15] of the format $2 \times 2 \times 2$ for the hypermatrix $A$ with 
components $a_{i j k}, i, j, k \in\{0,1\}$, is given by

$$
\begin{aligned}
\operatorname{Det}(A)= & a_{000}^{2} a_{111}^{2}+a_{001}^{2} a_{110}^{2}+a_{010}^{2} a_{101}^{2}+a_{100}^{2} a_{011}^{2} \\
& -2 a_{000} a_{001} a_{110} a_{111}-2 a_{000} a_{010} a_{101} a_{111} \\
& -2 a_{000} a_{011} a_{100} a_{111}-2 a_{001} a_{010} a_{101} a_{110} \\
& -2 a_{001} a_{011} a_{110} a_{100}-2 a_{010} a_{011} a_{101} a_{100} \\
& +4 a_{000} a_{011} a_{101} a_{110}+4 a_{001} a_{010} a_{100} a_{111}
\end{aligned}
$$

This hyperdeterminant can be written in a more compact form by using the Einstein convention and the Levi-Civita symbol $\varepsilon^{i j}$, with $\varepsilon^{00}=\varepsilon^{11}=0$, $\varepsilon^{01}=-\varepsilon^{10}=1$; and $b_{k n}=(1 / 2) \varepsilon^{i l} \varepsilon^{j m} a_{i j k} a_{l m n}, \operatorname{Det}(A)=(1 / 2) \varepsilon^{i l} \varepsilon^{j m} b_{i j} b_{l m}$. The four-dimensional hyperdeterminant of the format $2 \times 2 \times 2 \times 2$ has been given in Ref. [23].

In order to generate more invariants out of the hyperdeterminants, observe that the tensor product $I_{\mathbf{n}}=I_{d_{1}} \otimes \cdots \otimes I_{d_{n}}$ is a special hypermatrix of format $d_{1}^{2} \times \cdots \times d_{d}^{2}$ provided one views it as a tensor. Moreover for any $A=A_{1} \otimes \cdots \otimes A_{n} \in \hat{G}$, one has that

$$
\left(A_{1} *_{1} \cdots *_{n-1} A_{n} *_{n}\right) I_{\mathbf{n}}=A_{1} \otimes \cdots \otimes A_{n}
$$

which is also an element of $\operatorname{Mat}\left(d_{1}^{2}, \cdots, d_{n}^{2}\right)$.

Theorem 5 (1) The hyper-characteristic polynomial $\operatorname{Det}\left(\lambda I_{\mathbf{n}}-\rho\right)=\operatorname{Det}\left(\lambda I_{\mathbf{n}}-[\rho]\right)$ of the multipartite mixed state $\rho$ with $[\rho]=\left(A_{i_{1} \cdots i_{n}}\right) \in \operatorname{Mat}\left(d_{1}^{2}, \cdots, d_{n}^{2}\right)$, is a SLOCC-invariant polynomial in $\lambda$. The coefficients of the characteristic polynomial are all SLOCC-invariants. In particular, the hyper-trace $\operatorname{Tr}(A)$ and the hyperdeterminant $\operatorname{Det}(A)$ of $A$ are two distinguished polynomial SLOCC-invariants. (2) The same results hold for even-partite quantum states when Det is replaced with hdet.

Proof: Both hyperdeterminants are proved similarly, so we only consider Det. By previous discussion either the transition matrix or the action of the SLOCC-symmetry is represented by the hyper-matrix multiplication, see (3) and (9):

$$
\begin{aligned}
{[\rho]_{\sigma^{\prime}} } & =\left(P_{1} *_{1} \cdots *_{n-1} * P_{n} *_{n}\right)[\rho]_{\sigma}, \\
{\left[g \rho g^{\dagger}\right] } & =\left(B_{1} *_{1} \cdots *_{n-1} * B_{n} *_{n}\right)[\rho] .
\end{aligned}
$$


Therefore the hyper-characteristic polynomial of $g \rho g^{\dagger}$ is

$$
\begin{aligned}
& \operatorname{Det}\left(\left(B_{1} *_{1} \cdots *_{n-1} B_{n} *_{n}\right)\left(\lambda I_{\mathbf{n}}-[\rho]\right)\right) \\
& =\operatorname{det}\left(B_{1}\right)^{N / d_{1}} \cdots \operatorname{det}\left(B_{1}\right)^{N / d_{1}} \operatorname{Det}\left(\lambda I_{\mathrm{n}}-\rho\right) \\
& =\operatorname{Det}\left(\lambda I_{\mathrm{n}}-\rho\right) .
\end{aligned}
$$

This means that $f(\lambda)=\operatorname{Det}\left(\lambda I_{\mathbf{n}}-\rho\right)$ is a SLOCC-invariant polynomial and every $\lambda$ coefficients of $f(\lambda)$ are SLOCC-invariants. Note that the above calculation also proves the independence from base change.

We emphasize that in general the state $I_{\mathbf{n}}$ is not represented by a tensor product of the identity operators except for the special bipartite case. This means that although in formality our result resembles the usual theory of characteristic polynomial, but the result contains substantially more information in general and constitutes a theoretical method to generate non-trivial invariants for the mixed states.

As an application of our general method, we give a few examples.

Example 1. For two-qubit case, $A=\left(a_{i j}\right) \in M_{4}$. The followings are SLOCC-invariants.

$$
\begin{aligned}
& \operatorname{Tr}(A) ; \quad \operatorname{det}(A) ; \\
& m_{12}+m_{13}+m_{14}+m_{23}+m_{24}+m_{34} \\
& m_{234}+m_{134}+m_{124}+m_{234}
\end{aligned}
$$

where $m_{i j}$ etc are the principal minors, i.e. $m_{12}$ is the $(1,2)$-principal minor of $A$.

Example 2. When $\rho$ is a mixed state on $H=\bigotimes_{1}^{2 n} \mathbb{C}^{d}$, we will get $d^{2}$ SLOCC invariants from $h \operatorname{det}\left(\lambda I_{\mathbf{n}}-\rho\right)$. The four nontrivial coefficients of the following polynomial provides SLOCC-invariants for the mixed 4-qubit state.

$$
\begin{aligned}
& h \operatorname{det}(A-\lambda I)= \\
& \frac{1}{24} \sum_{\sigma \in S_{4}^{3}} \operatorname{sgn}(\sigma) \prod_{i=1}^{4}\left(A_{\sigma_{1}(i) \sigma_{2}(i) \sigma_{3}(i) \sigma_{4}(i)}-\lambda \delta_{\sigma_{1}(i) \sigma_{2}(i)} \delta_{\sigma_{3}(i) \sigma_{4}(i)}\right) .
\end{aligned}
$$

\section{CONCLUSION AND DISCUSSION}

We have introduced a general set of SLOCC-invariants for mixed multipartite states by using hyperdeterminants of the matrix representation in terms of orthonormal bases. We have shown that these invariants are independent of matrix representations. 
Our method is fundamentally different from previous studies on LU-invariants of pure states, and enjoys favorable property of independence from base change. In fact, our method shows that as long as the transition matrix belongs to $\mathrm{SL}(n)$ then the hypermatrix representation of the mixed state $\rho$ can deliver the same result. Even in the case of pure quantum states, our method is also different. Suppose that a pure state is given by $|\phi\rangle=\sum_{I} a_{I}|I\rangle$, and assume that the transition matrix from $|I\rangle\langle J|$ to $\lambda$-basis is $\left(p_{I J, \sigma}\right)$, then the hypermatrix of $\rho=|\phi\rangle\langle\phi|$ is $\left(\sum_{I J} a_{I} a_{J}^{*} p_{I J, \sigma}\right)$, whose hyperdeterminant is quite different from that of $\left(a_{I}\right)$, even in the format.

The method of hyperdeterminants can be used for classification of multipartite quantum states besides theoretical classification of SLOCC symmetry. Since the invariants involve with large amount of computation, in practice they should be useful with the help of computer codes. In our opinion one of the useful messages is that many LU-invariants obtained from the hypermatrix representation are actually SLOCC-invariants.

\section{Acknowledgments}

NJ gratefully acknowledges the partial support of Simons Foundation, Humbolt Foundation, NSF, NSFC and MPI for Mathematics in the Sciences in Leipzig during this work.

[1] Gour G and Wallach N R, 2013, Phys. Rev. Lett. 111, 060502; 2010, J. Math Phys. 51, 112201.

[2] Yu N, Qiao Y and Sun X, Characterization of multipartite entanglement, arXiv:1401.2627.

[3] Albeverio S, Cattaneo L and Persio D L, 2007, Rep. Math. Phys. 60, 167.

[4] Fei S M and Jing N, 2005, Phys. Lett. A 342, 77.

[5] Grassl M R, Rötteler M and Beth T, 1998, Phys. Rev. A. 58, 1833.

[6] Rains E M, 2000, IEEE Trans. Inf. Theory. 46, 54.

[7] Makhlin Y, 2002, Quant. Info. Proc. 1, 243.

[8] Linden N, Popescu S and Sudbery A, 1999, Phys. Rev. Lett. 83, 243.

[9] Albeverio S, Cattaneo L, Fei S M and Wang X H, 2005, Int. J. Quant. Inform. 3, 603.

[10] Albeverio S, Fei S M and Goswami D, 2005, Phys. Lett. A. 340, 37.

[11] Albeverio S, Fei S M, Parashar P and Yang W L, 2003, Phys. Rev. A. 68, 010303.

[12] Sun B Z, Fei S M, Li-Jost X Q and Wang Z X, 2006, J. Phys. A. 39, 43. 
[13] Kraus B, 2010, Phys. Rev. Lett. 104, 020504; 2010, Phys. Rev. A 82, 032121.

[14] Liu B, Li J L, Li X and Qiao C F, 2012, Phys. Rev. Lett. 108, 050501.

[15] Cayley A, 1845, Cambridge Math. J. 4, 193.

[16] Gelfand I M, Kapranov M M and Zelevinsky A V, 1994, Discriminants, Resaultants, and Multidimensional Determinants. Birkhaeuser, Boston.

[17] Albeverio S and Fei S M, 2001, J. Opt. B: Quantum Semiclass. Opt. 3, 223.

[18] Hill S and Wootters W K, 1997, Phys. Rev. Lett. 78, 5022.

[19] Rungta P, Buzek V, Caves C M, Hillery M and Milburn G J, 2001, Phys. Rev. A. 64, 042315.

[20] Uhlmann A, 2000, Phys. Rev. A. 62, 032307.

[21] Wootters W K, 1998, Phys. Rev. Lett. 80, 2245.

[22] Coffman V, Kundu J and Wootters W K, 2000, Phys. Rev. A. 61, 052306.

[23] Luque J G and Thibon J Y, 2003, Phys. Rev. A. 67, 042303.

[24] Miyake A, 2003, Phys. Rev. A. 67, 012108.

[25] Viehmann O, Eltschka C and Siewert J, 2011, Phys. Rev. A 83, 052330.

[26] Zhang T G, Jing N, Li-Jost X Q, Zhao M J and Fei S M, 2013, Euro. Phys. J. D 67, 175.

[27] Horn R A and Johnson C R, 1994, Topics in matrix analysis. Cambridge University Press, Cambridge. 